\title{
Antenna Solutions for 4G Smartphones in Laser Direct Structuring Technology
}

\author{
Aykut CIHANGIR ${ }^{1}$, Florence SONNERAT ${ }^{2}$, Frederic GIANESELLO ${ }^{2}$, Daniel GLORIA ${ }^{2}$, \\ Cyril LUXEY ${ }^{1}$ \\ ${ }^{1}$ EpOC, Université Nice-Sophia Antipolis, 650 Route des Colles, 06560 Valbonne, France \\ ${ }^{2}$ STMicroelectronics, 850 Rue Jean Monnet, 38920 Crolles, France \\ cyril.luxey@unice.fr
}

Manuscript received August 1, 2016

\begin{abstract}
Two antenna designs operating in the LTE/4G cellular frequency bands are proposed in this paper. Both designs consist of a driven strip which capacitively excites a parasitic grounded strip for a better matching response around $700 \mathrm{MHz}$. The antennas are realized using Laser Direct Structuring (LDS) technology on POCAN plastic material with a height of $5 \mathrm{~mm}$ above the system Printed Circuit Board. Passive matching circuits are necessary at each antenna's feed, to match the input impedance to $50 \mathrm{Ohm}$ at the desired operating bands $(700-960 \mathrm{MHz}$ and 1.7-2.2 GHz for the first design and 700-960 MHz and 1.7-2.7 GHz for the second). The simulation results are validated through s-parameter and total efficiency measurements. To form a basis for future studies, the effect of the hand and the head of the user are investigated upon the antenna performance.
\end{abstract}

\section{Keywords}

Mobile antennas, parasitic antennas, LTE, 4G, LDS, matching network

\section{Introduction}

The cellular mobile technology has undergone several improvements in the past decades. The first cellular communication systems were introduced around the $80 \mathrm{~s}$ as analog FM systems [1]. The modulation schemes were changed to digital around the $90 \mathrm{~s}(2 \mathrm{G})$ and evolutions containing new specifications like Short Message Services (SMS) and low-speed data services like GPRS (2.5G General Packet Radio Service) and EDGE (2.75G - Enhanced Data rates for GSM Evolution) were quickly introduced. Approximately ten years later, with the customer demands for more data exchanges with the introduction of smartphones, third generation of cellular communications started to provide extremely fast internet access using technologies like HSPA (High Speed Packet Access). With the never-ending demand for higher data rates, $4 \mathrm{G}$ was intro- duced using the LTE-A (Long-Term Evolution Advanced) standard which targets to support as high as $100 \mathrm{Mbps}$ for high mobility and up to $1 \mathrm{Gbps}$ for stationary user ends. The new specifications introduced with LTE-A are mainly the true exploitation of MIMO (Multiple-Input MultipleOutput) at both the base station and the user-end sides, frequency carrier aggregation, enlargement of the carrier bandwidth up to $20 \mathrm{MHz}$ and newly added frequency bands like bands the LTE7, 12, 13 etc.

From the point of view of the antenna designer, the new specifications reveal the necessity for the mobile terminal antenna to cover the $700-960$ and $1700-2700 \mathrm{MHz}$ frequency bands. The biggest challenge is to cover a relatively large bandwidth of $30 \%$ in the Low-Band (LB) since the physical space reserved for the antenna in a typical mobile terminal is electrically small compared to the freespace wavelength at $700 \mathrm{MHz}$. For this purpose, Coupling Elements (CEs) were proposed in [2], [3] which serve as simple structures to capacitively excite the currents on the ground plane of the Printed Circuit Board (PCB). In this way, the low-quality factor of the wide ground plane, whose $100 / 120 \mathrm{~mm}$ length typically corresponds to a halfwavelength at a frequency close to respectively $1.15 / 1.00 \mathrm{GHz}$, is exploited. The antenna impedance is then tuned to cover the desired frequency band with the help of a proper Matching Network (MN). In [2], two CEs with their MNs were placed on the short edge of the ground plane of the PCB, one being responsible for the GSM band coverage $(824-960 \mathrm{MHz})$ and the other for the DCS/PCS band coverage (1.71-1.99 GHz). Special attention was paid for the LB MN to get a high impedance behavior at the high-band (HB) and vice-versa. The two MN branches were then simply combined together obtaining a single-feed antenna. In [3], a dual-CE configuration called ground plane boosters was proposed with the two $\mathrm{MN}$ branches being connected after using notch-filters in each branch. In [4], [5], antennas were proposed to enlarge the bandwidth coverage down to $700 \mathrm{MHz}$ (low-LTE band). The antenna in [4] may operate in the low LTE and UMTS frequency bands (700-960 MHz and 1.7-2.2 GHz) utilizing a single $\mathrm{CE}$. The matching circuit at the feed con- 
sists of a two-branched $\mathrm{MN}$ for dual-band operation, having nine SMD components. In [5], the operating frequency was extended down to $700-960 \mathrm{MHz}$ and up to 1.7-2.7 GHz, covering the $4 \mathrm{G}$ cellular communication bands. The antenna acts as a dual-feed structure where the LB feed uses the radiating element as a CE whereas the HB feed (having an offset in excitation point compared to the LB feed) is using also the resonant properties of the radiating element. These two feeds are then combined together to obtain a single feed access after introducing a two branch MN of eight lumped components where each branch behaves as an open circuit regarding the other. The main advantage of this type of antennas is the high bandwidth potential due to the utilization of the low-Q property of the ground plane of the PCB (thick radiating structure). However, dual-band behavior is tricky to obtain, especially with a single-feed solution: it requires a very special care for the design of the matching networks. Also, the number of SMD (Surface Mounted Devices) components are relatively high since a complicated $\mathrm{MN}$ is required which in turn increases both the losses due to the internal resistances and also the overall cost of the antenna. An alternative method is to use a parasitic element for bandwidth enlargement, like in [6], [7], where a driven monopole strip is used to excite a longer parasitic element connected to the ground plane of the PCB on one (or both) edge(s) of this ground plane. In this kind of antennas, the LB coverage is mainly achieved by the capacitive excitation of the parasitic element and the $\mathrm{HB}$ is covered using the driven monopole and the higher order resonances of the parasitic strip. The antennas presented in [8-11] use the same strategy to obtain 4G LTE coverage in mobile terminal antennas, taking into account also a dielectric casing with loss tangents varying between $0.01-0.02$. Due to dielectric loading effect and losses, the casing makes it easier for the antenna to operate down to $700 \mathrm{MHz}$ especially shifting its resonance to lower frequencies. In [12], [13], similar antenna topologies using dielectric casings were presented. Although they are called Planar-Inverted-F Antennas (with coupled feeds), the main strategy is the same as using a shorter driven strip which capacitively excites a longer parasitic strip connected to the ground plane of the PCB. The possible drawback of these antennas is the relatively complicated design and complicated antenna layout which may be a problem when the manufacturing tolerances are of concern.

Therefore, the main target of our study is to design a generic antenna that can be easily integrated in every smartphone. As an obvious observation, a common part in every smartphone is the touch screen. However, they are quite different with quite various dimensions from phone to phone. The shortest available 3.5" touchscreen was chosen here as the ground plane of the PCB to emulate a usecase being a worst-case condition in terms of bandwidth potential (It can be foreseen that the bandwidth potential of this worst-case smartphone will be subject to improve if a longer ground plane is used by the manufacturer). The half-resonance of such a short $75 \mathrm{~mm}$ ground plane would occur approximately at $1.4 \mathrm{GHz}$, which is far away from the lowest targeted frequency in the LB which makes it really hard to be exploited with a simple CE. So far, from our best knowledge, such an approach with those hard constraints has never been tempted before for smartphones. As a simple CE might not be sufficient for proper operation, it was intended to use a shorted parasitic element capacitively excited by a driven strip to ease LB frequency coverage, whereas the HB coverage is mainly achieved by the driven strip. To achieve this goal, a novel point is the realization of the antennas using Laser Direct Structuring (LDS) technology, since a 3D antenna approach is absolutely necessary to maximize the allocated volume to the antenna structure. Using this technology, a high degree of freedom is obtained in terms of metallization shape (3D regarding the chosen plastic piece), thus enabling creativity to improve radiation performance.

In Sec. 2 of this paper, we validate the novel proposed design approach in free space with an antenna concept that can cover the $700-960 \mathrm{MHz}$ frequency band as the LB and the 1.7-2.2 GHz (DCS/PCS/UMTS) frequency band as the HB. In Sec. 3, an improved antenna design that operates in the $700-960 \mathrm{MHz}$ and $1.7-2.7 \mathrm{GHz}$ frequency bands is presented. The S-parameters and total efficiency measurements including a hand and head phantom to account for the user effect are also presented in Sec. 4. The paper is then finalized with a conclusion.

\section{First LDS Antenna Design}

As already mentioned, a simple CE is not sufficient to establish broad dual-band frequency coverage in the targeted LB and HB especially if the length of the ground plane is $75 \mathrm{~mm}$. Therefore, a novel design which consists of a driven strip which capacitively excites a grounded parasitic strip is introduced in Fig. 1.

The parasitic strip (shown in red in Fig. 1), optimized for a reflection coefficient staying below $-6 \mathrm{~dB}$ in the target bands, and the driven strip (shown in orange in Fig. 1) are printed on a Pocan DP T7140 [14] plastic piece (shown in grey in Fig. 1) using the LDS technology. As also described in [15], the LDS process uses a thermoplastic material which has already been doped with a metal-plastic additive. A laser beam tracks the surface of the material, according to the shape of the traces intended on it. In this way, a microscopically rough surface is created, forming the nuclei for the metallization step. Finally, the metal traces are formed by putting the laser activated material in the electroless copper baths. The relative permittivity and loss tangent of the plastic material are given as 4.1 and 0.0138 by the manufacturer, at $1 \mathrm{MHz}$. Although not very optimal in terms of electromagnetic properties, this material was chosen since it allows reflow soldering which enables to integrate components directly on the material as presented in [16]. The parasitic strip is connected to the ground plane (under the PCB substrate) using a via passing through the FR4 substrate. There is a matching network at 


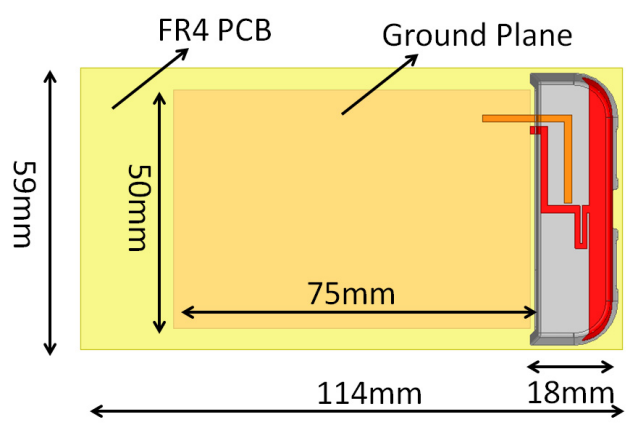

(a)

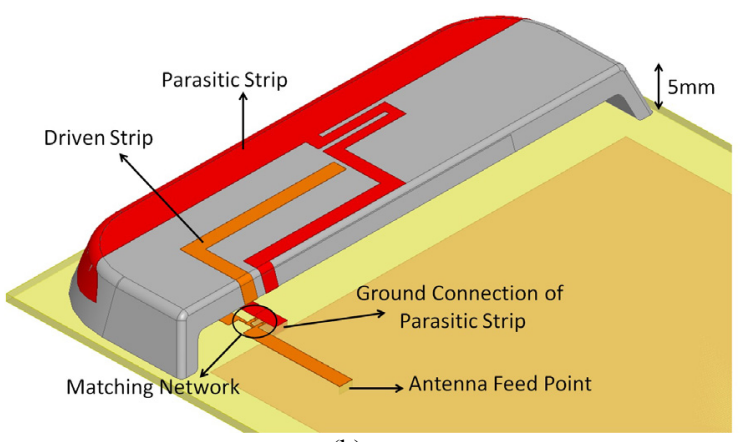

(b)

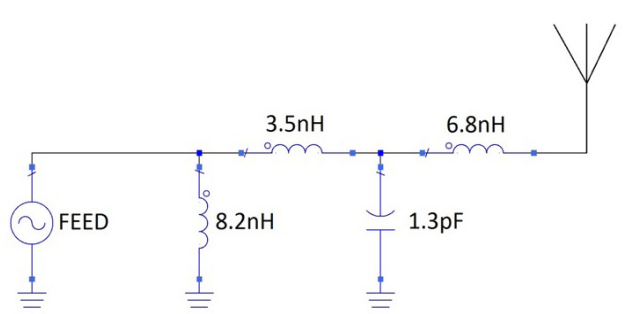

(c)

Fig. 1. Simulation Model of the first antenna. (a) Top view of the PCB and the antenna. (b) Close view of the plastic piece. (c) Matching network values.

the antenna feed, composed of four lumped components chosen as 0201 SMD package (Fig. 1c). The ground plane of the system has a length and width of $75 \times 50 \mathrm{~mm}^{2}$ whereas the total PCB dimensions are $114 \times 59 \times 0.8 \mathrm{~mm}^{3}$ (Fig. 1a). The connection between the traces on the plastic piece and the traces on the PCB are achieved using two pogo-pins under the plastic piece.

The simulated input impedance of the antenna without the $\mathrm{MN}$ is shown in Fig. 2, plotted for LB (between 700-960 MHz, black plain line) and for HB (1.7-2.2 GHz, black dotted line). The benefit of inserting the parasitic strip can be seen when the input impedance is compared with the case of an antenna with a driven strip only (orange strip in Fig. 1b and red curves in Fig. 2). With the introduction of the parasitic strip (black plain curve), a new resonance is created in the $\mathrm{LB}$, which is seen as a loop locus in the Smith chart. The length of the parasitic strip was adjusted by inserting a meandered section to get enough bandwidth potential (BP) in the center of the LB. Adding the parasitic strip increases considerably the real part of the input impedance in the LB. In this way, the

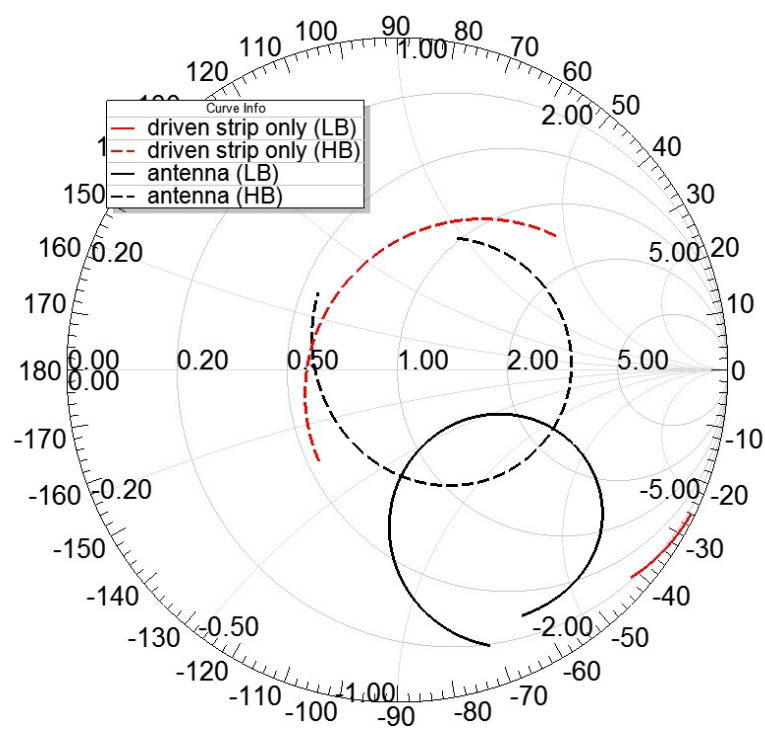

Fig. 2. Simulated input impedance versus frequency (LB and HB) of the driven strip only (red curves) and of the first optimized antenna without MN (black curves).

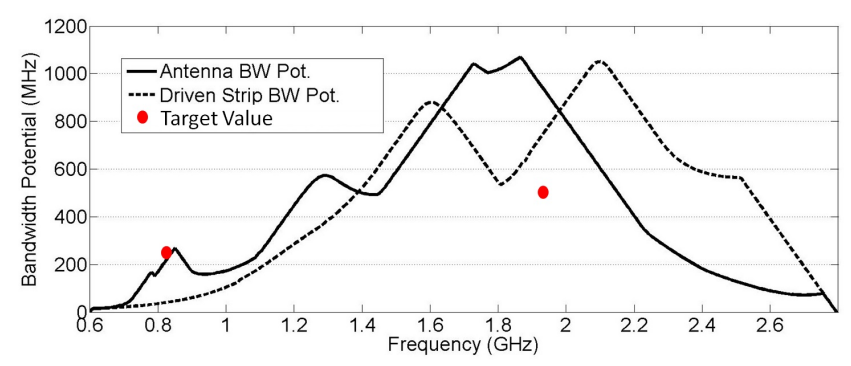

Fig. 3. Bandwidth potential of the first antenna with/without parasitic strip.

obtainable BP is considerably increased (from $45 \mathrm{MHz}$ to $250 \mathrm{MHz}$, Fig. 3) especially in LB whereas no significant $\mathrm{BP}$ change is observed in the $\mathrm{HB}$, only a small frequency shift of the HB resonances. It is important to explain that the BP presented in Fig. 3 is a realistic value (rather than a theoretical too much optimistic value) computed by Optenni Lab software [17]. This BP value is obtained by optimizing a $\mathrm{MN}$ of two components for every frequency point and extracting from the resulting input impedance the frequency interval which is under a threshold reflection coefficient, chosen as $-6 \mathrm{~dB}$ here. At last, Figure 3 indeed shows that the driven strip alone cannot meet the LB specifications (the target BP values are shown with the two red dots) and therefore, the necessity of using the parasitic strip for bandwidth enlargement to meet those target specifications.

The effect of the parasitic strip can also be observed when looking at the surface currents induced on the antenna and the ground plane of the PCB at 750 and $900 \mathrm{MHz}$ (Fig. 4). The induced currents on the parasitic strip are stronger at $750 \mathrm{MHz}$ than $900 \mathrm{MHz}$. The currents on the ground plane are excited in approximately the same way at both frequencies: flowing in the longitudinal direction regarding the length of the PCB. 


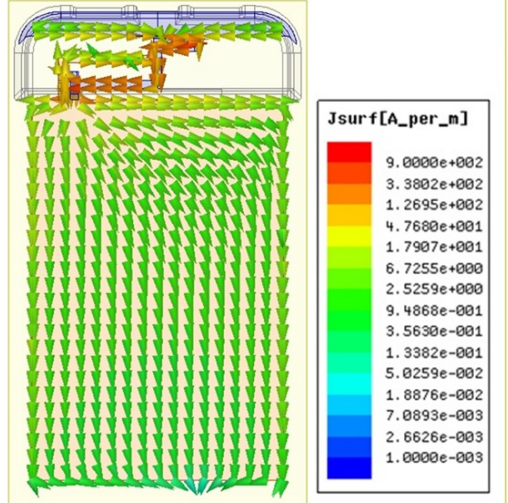

at $750 \mathrm{MHz}$

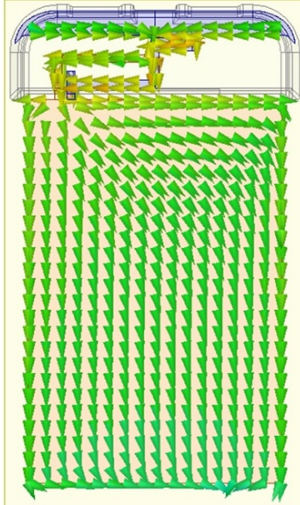

at $900 \mathrm{MHz}$

Fig. 4. Surface currents of the antenna and the ground plane of the PCB presented in Fig. 1 at $750 \mathrm{MHz}$ and $900 \mathrm{MHz}$

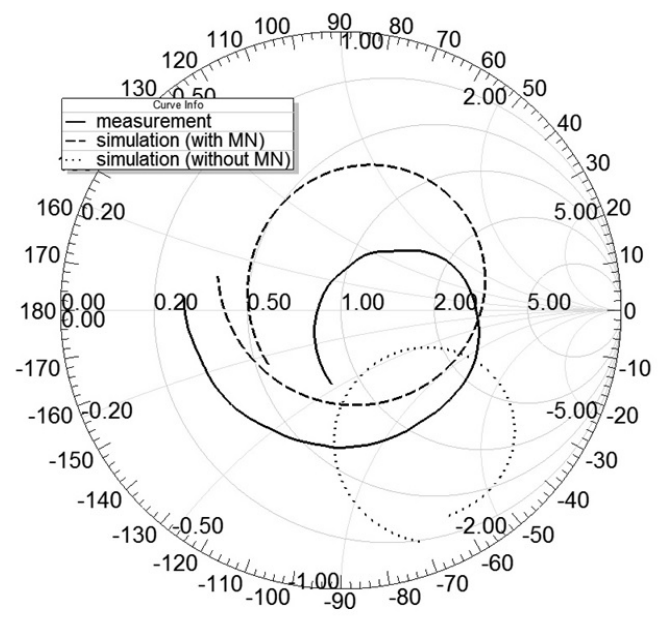

LB $(700-960 \mathrm{MHz})$

(a)

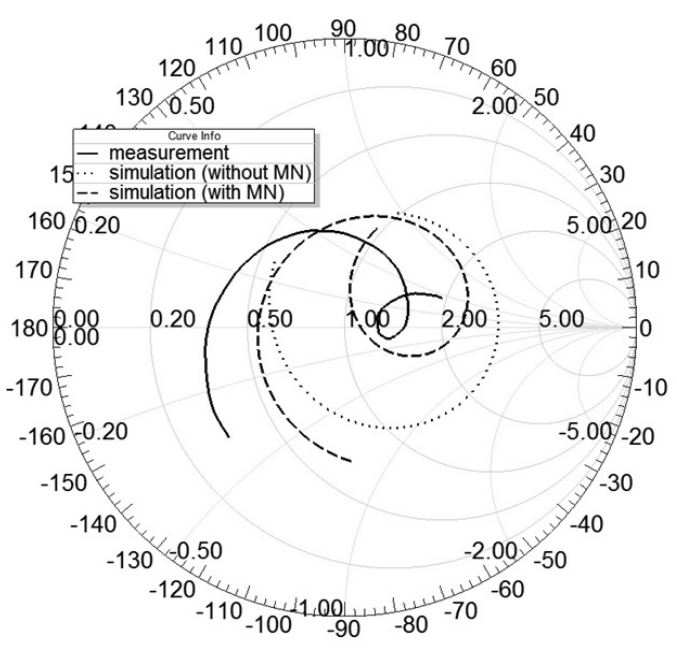

$\mathrm{HB}(1.7-2.2 \mathrm{GHz})$

(b)

Fig. 5. Simulated and measured input impedance of the antenna presented in Fig. 1: (a) LB, (b) HB.

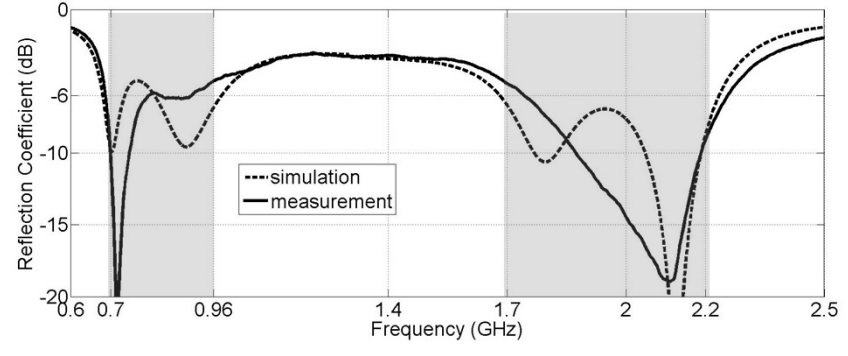

Fig. 6. Simulated and measured reflection coefficient of the antenna presented in Fig. 1.

The simulated input impedance with/without the MN can be observed in Fig. 5. The measured input impedance of the manufactured prototype is also presented. To account for the losses of the SMD components, the corresponding ESRs (Equivalent Series Resistance) were also included in the full electromagnetic simulations (HFSS). Generally, a fair agreement can be seen between the simulated input impedances with $\mathrm{MN}$ and the measured input impedances. The antenna indeed covers the target bands with a reflection coefficient below $-6 \mathrm{~dB}$ except some overshoots close to the edges of the target bands rising up to $-5 \mathrm{~dB}$ (Fig. 6). One main reason about the small difference between simulation and measurement can be the unknown characteristics of the Pocan material, since the electrical properties at $1 \mathrm{MHz}$ are used for modeling. The manufactured antenna prototype can be seen in Fig. 7 alone and also in the Satimo Starlab station for radiation pattern and total efficiency measurements. The antenna is fed through an SMA connector placed on the PCB. A standard calibration procedure is used. In free-space, two configurations were measured, the first one with the original $75 \mathrm{~mm}$ ground plane and the other with an extended ground plane of $90 \mathrm{~mm}$ using copper tape. No significant change of the measured reflection coefficient was observed for the two different ground plane lengths. The antenna with short ground plane has a total efficiency higher than $-4 \mathrm{~dB}$ in $70 \%$ of the LB frequency range but rather low at $700 \mathrm{MHz}$ ( $-7.7 \mathrm{~dB}$ in Fig. 8). When the length of the ground plane is extended to $90 \mathrm{~mm}$, there is an increase in the total efficiency: it is above $-3 \mathrm{~dB}$ in $70 \%$ of the LB range and it stays always higher than $-4.3 \mathrm{~dB}$. The HB efficiency stays between $-2 \mathrm{~dB}$ and $-4 \mathrm{~dB}$, regardless the length of the ground plane. It should be noted that the $\mathrm{MN}$ of the antenna prototype with a $90 \mathrm{~mm}$ ground plane was not retrooptimized. The radiation pattern has an omni-directional

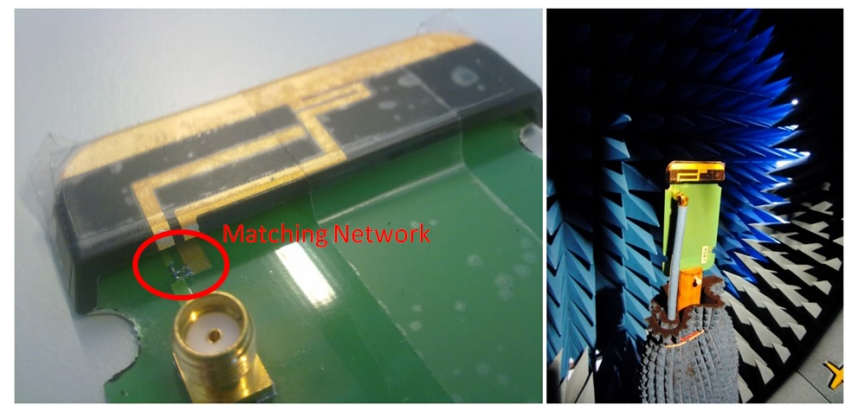

Fig. 7. Manufactured antenna and measurement environment. 


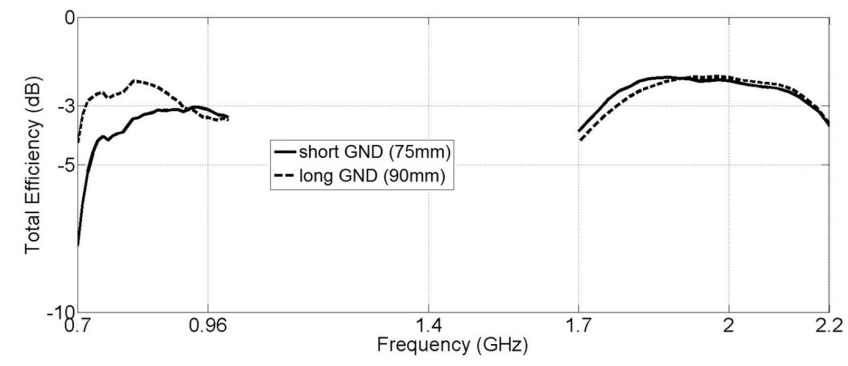

Fig. 8. Measured total efficiency of the antenna presented in Fig. 1 (short GND) and the same antenna with extended ground plane to $90 \mathrm{~mm}$ (long GND).

shape in the LB, being more directive as the frequency increases in the HB, as expected.

At this stage, the design approach seems to be validated in terms of obtainable matching and total efficiency performance for an internal antenna in LDS technology. This antenna concept is suitable for a mobile terminal with a short generic ground plane. The next goal is to design the same kind of antenna for LTE/4G purpose, i.e. covering 700-960 MHz and 1.7-2.7 GHz.

\section{LTE Antenna Design}

Using the same methodology, another antenna seen in Fig. 9 was designed and optimized to be able to cover the low and high-LTE frequency bands. The main idea was to obtain an antenna with enough BP in the LB and already good matching in the $\mathrm{HB}$ without any MN. Then, with an appropriate $\mathrm{MN}$ topology, the LB is matched without too much affecting the HB matching. The PCB and ground plane dimensions were kept the same. The antenna consists again of a driven strip which capacitively excites a grounded parasitic strip. The orientation of the HB driven strip was changed when compared to the previous design, with the open-end facing the long corner of the ground plane. This orientation has shown more bandwidth potential in the $\mathrm{HB}$, enabling the simultaneous coverage of $0.7-0.96 \mathrm{GHz}$ and $1.7-2.7 \mathrm{GHz}$. This configuration has also enabled reducing the number of SMD components to two in the matching network (Fig. 9c) instead of four in the previous design. However, the shunt inductor was split into two inductors for series resonance frequency issues, which finally makes a total of three SMD components in the final prototype.

The simulated input impedance of the antenna without the MN is shown in Fig. 10 (black curves), plotted for LB between $700-960 \mathrm{MHz}$ (plain line) and for $\mathrm{HB}$ between 1.7-2.7 GHz (dotted line). The benefit of inserting the parasitic strip can be seen when the input impedance is compared with the case of an antenna with a driven strip only (orange element in Fig. 9 and red plain and dotted lines in Fig. 10). With the introduction of the parasitic strip (black plain curve), a new resonance is created in the LB which is seen as a locus loop in the Smith chart. In fact, adding the parasitic strip increases considerably the real part of the input impedance in the LB. In this way, the

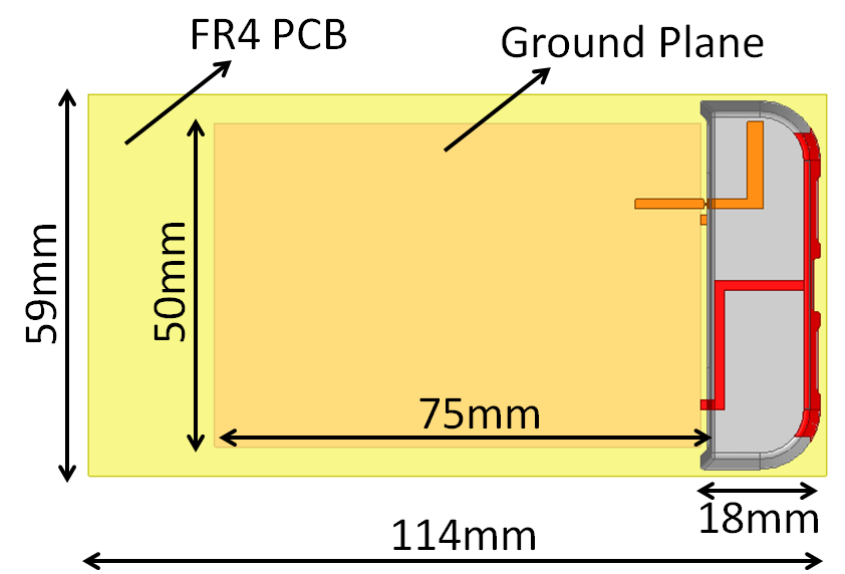

(a)

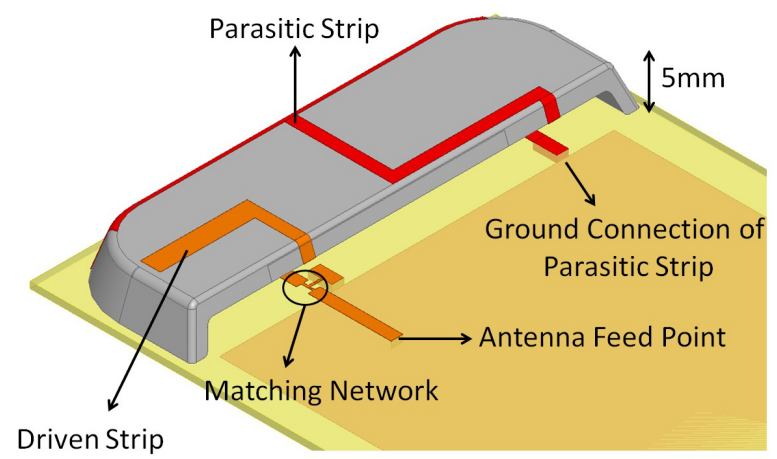

(b)

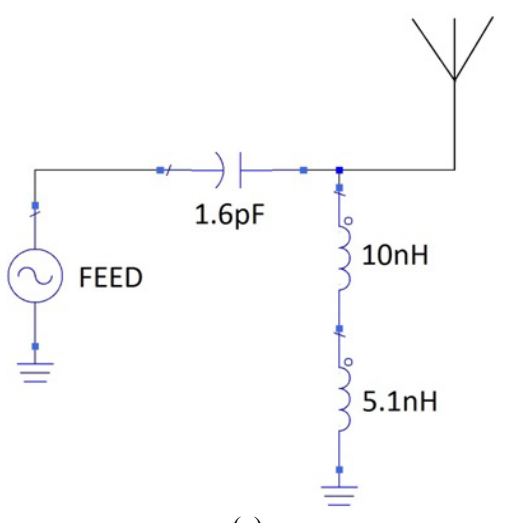

(c)

Fig. 9. Simulation model of the LTE antenna. (a) Top view of the PCB and the antenna. (b) Close view of the plastic piece. (c) Matching network.

obtainable BP is considerably increased, as much as $370 \%$, especially in LB whereas a slight BP increase is also observed in the HB (Fig. 11). The effect of the parasitic strip in the HB can be seen in Fig. 12. Strong currents can be observed on the parasitic strip at $2 \mathrm{GHz}$, corresponding to the resonance seen as the loop of the input impedance. At $2.5 \mathrm{GHz}$, the driven strip is strongly excited whereas there are weak currents on the parasitic strip.

The simulated and measured input impedance with the MN can be seen in Fig. 13. Looking at Fig. 14, it can be told that the LB frequency range is covered with a reflection coefficient below $-6 \mathrm{~dB}$ except some overshoots close to the higher edge of the band. In the HB, the match- 


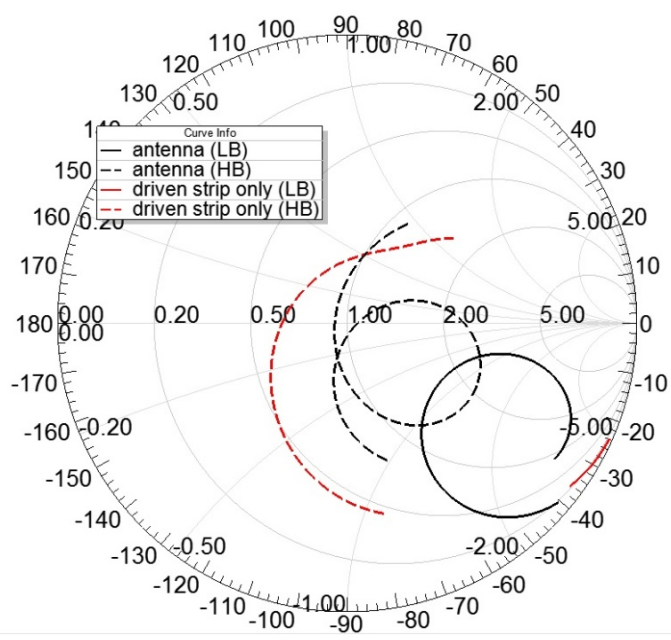

Fig. 10. Simulated input impedance versus frequency (LB and $\mathrm{HB})$ of the driven strip only (red curves) and of the LTE optimized antenna without MN (black curves).

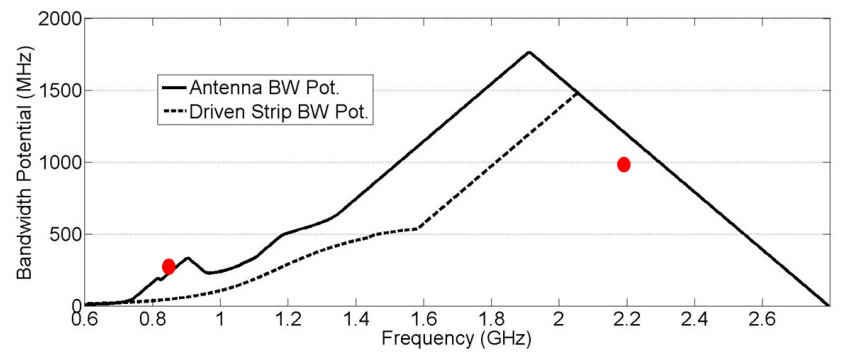

Fig. 11. Bandwidth potential of the LTE antenna with/without parasitic strip.

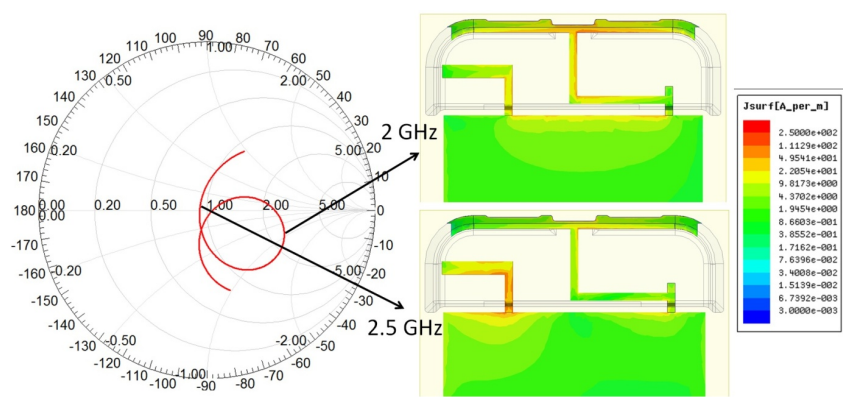

Fig. 12. Effect of the parasitic strip on the HB input impedance.

ing is poor around $1700 \mathrm{MHz}$ but except this region, the whole LTE HB is covered with a reflection coefficient below $-6 \mathrm{~dB}$. There is a fair agreement between simulation and measurement results.

The total efficiency measurements were achieved for the manufactured prototype presented in Fig. 15. Same SMA connector and feed cable placements were used as the previous antenna measurement. For the short ground plane configuration, the total efficiency is higher than $-5 \mathrm{~dB}$ in $70 \%$ of the $\mathrm{LB}$, rising up to a maximum of $-3 \mathrm{~dB}$ (Fig. 16), with omnidirectional radiation pattern. By increasing the length of the ground plane until $90 \mathrm{~mm}$, no significant changes were observed in the reflection coefficient (without matching network optimization) but a significant increase was observed in the total efficiency becoming higher than $-4 \mathrm{~dB}$ in $70 \%$ of the band with

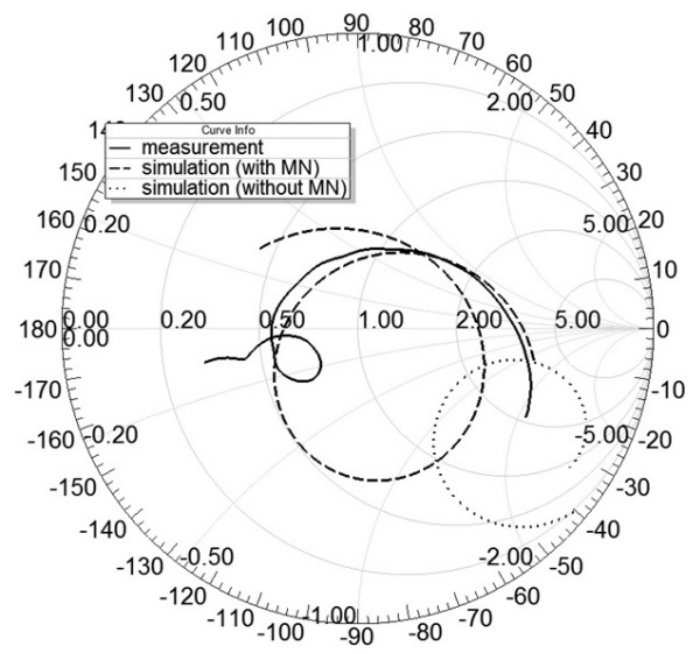

LB $(700-960 \mathrm{MHz})$

(a)

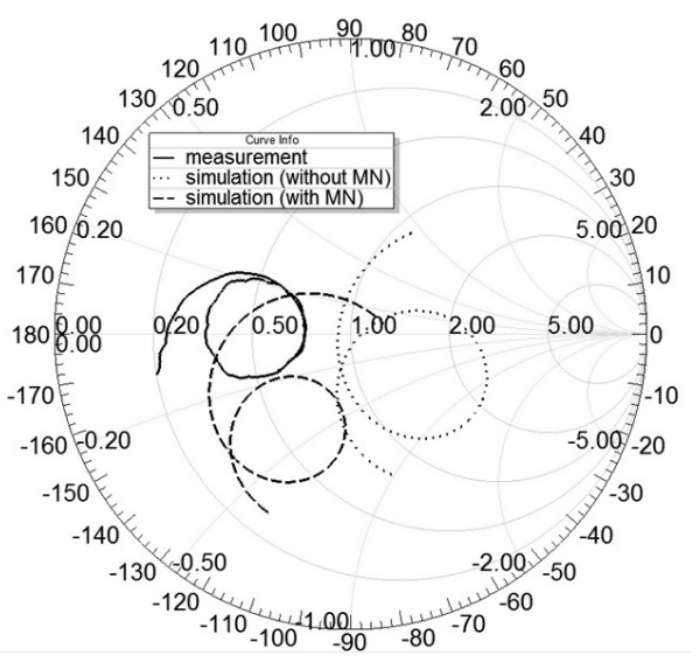

$\mathrm{HB}(1.7-2.7 \mathrm{GHz})$

(b)

Fig. 13. Simulated and measured input impedance of the LTE antenna: (a) LB, (b) HB.

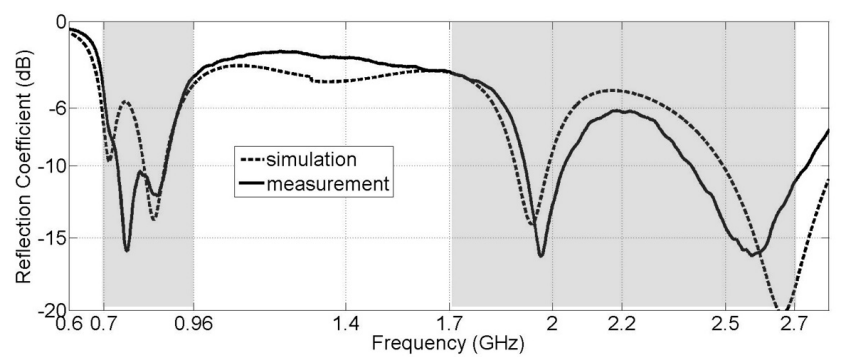

Fig. 14. Simulated and measured reflection coefficient of the LTE antenna.

a maximum peak of $-2.3 \mathrm{~dB}$. Like the previous prototype, the length of the ground plane has no significant impact on the HB total efficiency. Due to the poor matching around $1700 \mathrm{MHz}$, a lower efficiency is observed around the lowedge of the HB. Except this interval, the measured total efficiency remains between $-3.5 \mathrm{~dB}$ and $-2 \mathrm{~dB}$. 


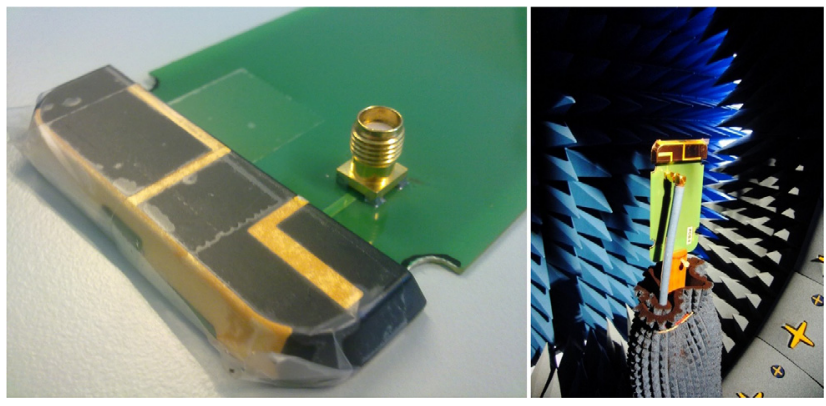

Fig. 15. Manufactured LTE antenna and measurement environment.

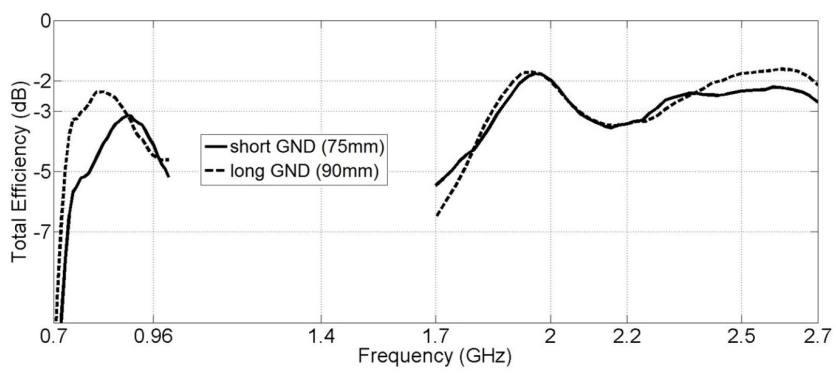

Fig. 16. Measured total efficiency of the LTE antenna (short GND) and the same antenna with the extended ground plane to $90 \mathrm{~mm}$ (long GND).
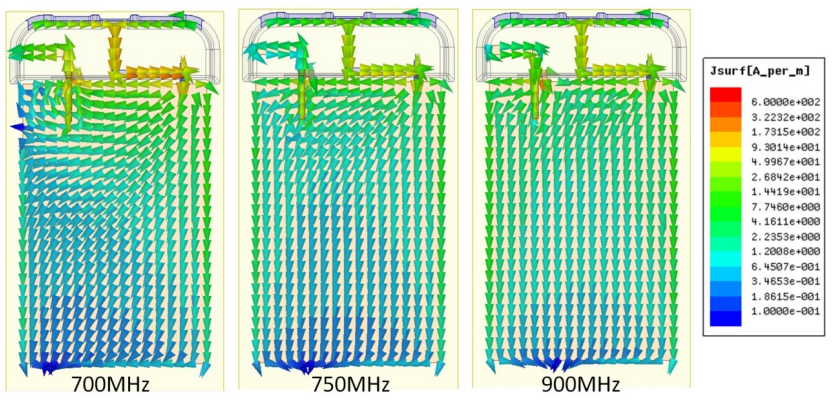

Fig. 17. Surface current distribution on the LTE antenna and ground plane in the LB.

To investigate the reason of the low total efficiency at $700 \mathrm{MHz}$ despite reasonable reflection coefficient, the surface currents were plotted at three different frequency points (Fig. 17). At $700 \mathrm{MHz}$, strong excitation of the parasitic strip is seen where the currents circulate following the path: feed-driven strip-parasitic strip-short edge of ground plane-back to the feed. For this reason, the currents on the system ground plane (especially on the long edges) are weak when compared to more uniform excitation in the longitudinal direction at $750 \mathrm{MHz}$ and $900 \mathrm{MHz}$. Since most of the radiation comes from the first chassis wavemode in this frequency range, the low radiation efficiency can be explained by the currents circulating between the driven strip and parasitic strip and not forming the chassis wavemode.

\section{Investigation of the User Effect}

The design of an internal mobile phone antenna operating in the LTE bands in LDS technology placed over a short generic ground plane has been validated but our design approach was to optimize matching and total efficiency performance in free space. In this work, there was no special intention on designing the antennas considering the user effects.

To form a basis for future studies, both s-parameter and efficiency were measured using a hand and a head phantom, to see the effects of the user on these optimallyovercoupled antenna prototypes. First, to obtain a more realistic mock-up, a dielectric casing that is made of ABSP430 material with relative permittivity of 2.4 and a loss tangent of 0.0053 (at $60 \mathrm{MHz}$ ) was used to house the antenna and its PCB. The thickness of the casing is $1 \mathrm{~mm}$ on the top and $3 \mathrm{~mm}$ at the bottom of the PCB. In all the measurements, the antenna was placed at the bottom side of the casing, facing the palm. The measurement set-up using the phantom hand and head can be observed in Fig. 18.

It is well known that bringing the user's hand in front of an antenna will result in shifting its resonance frequency

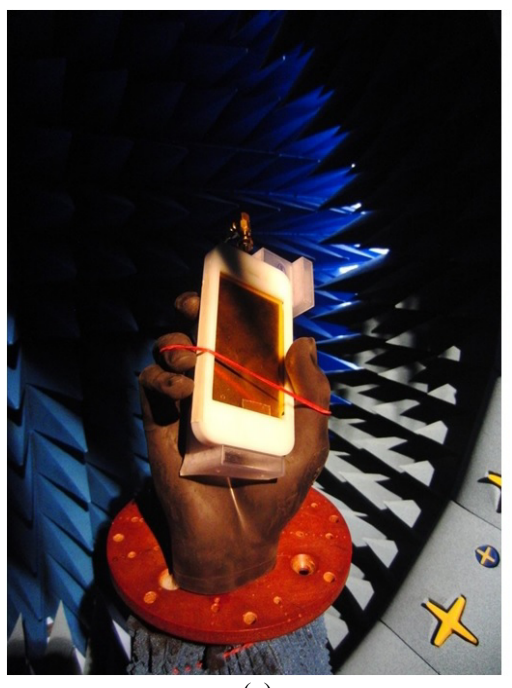

(a)

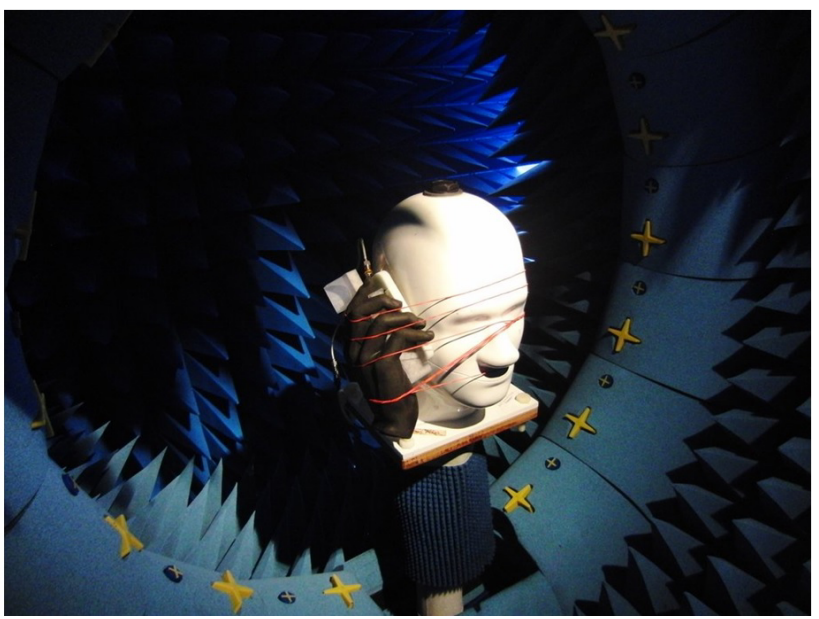

(b)

Fig. 18. Measurement setup with user effects: (a) hand phantom configuration, (b) hand \& head phantom configuration. 


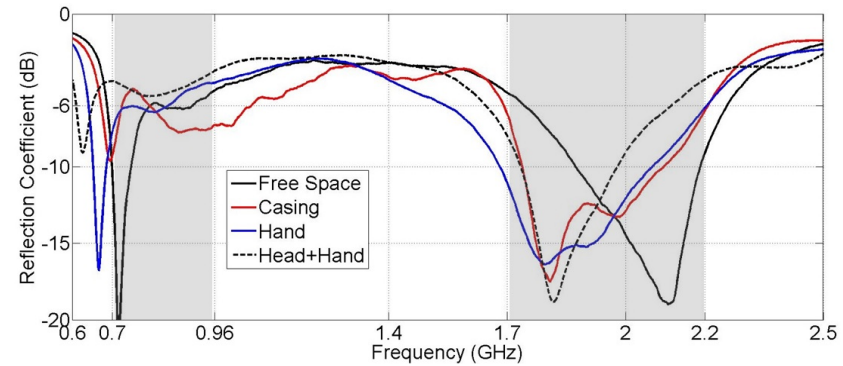

Fig. 19. Measured reflection coefficient of the first antenna design taking into account the user effects.

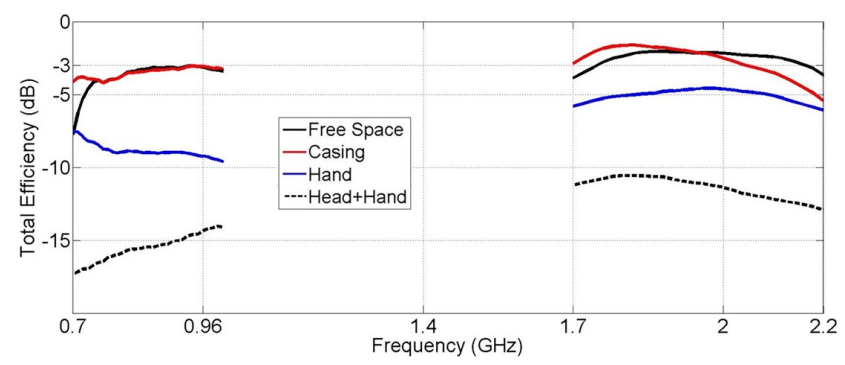

Fig. 20. Measured total efficiency of the first antenna taking into account user effects.

down, due to dielectric loading as well as losses, this last effect being difficult to overcome. This effect is seen in Fig. 19 for the first antenna presented in Sec. 2. With the introduction of the hand and head suitably positioned with the handset, a shift in the resonance frequency can be seen towards the lower frequencies. The effect is dominant in the LB (observed as detuning) whereas it can be neglected in the HB. The measured total efficiency for the four cases (free space, with casing, with casing and hand, with casing, hand and head) is presented in Fig. 20. It is observed that introducing the casing around the antenna had a slightly positive effect on the efficiency through dielectric loading. The resonance created by the parasitic strip around $700 \mathrm{MHz}$, which has lower efficiency than other frequencies since the currents are now concentrated mainly on the parasitic strip (ground plane is weakly excited), is shifted down which increases the radiation efficiency and thus the total efficiency in the lower part of the LB (between $700 \mathrm{MHz}$ and $730 \mathrm{MHz}$ ). When the hand phantom is taken into account, there is approximately a $5 \mathrm{~dB}$ decrease in the total efficiency making it below $-8 \mathrm{~dB}$ due to both detuning effects and body-loss mechanisms. The same behavior can also be observed in HB where the efficiency drops to the $-5 \mathrm{~dB}$ level due to losses in the hand. As expected, the radiation pattern is also modified now with a radiated beam mainly pointing in the direction not covered by the hand. When the head is also taken into account, further decrease is observed in the measured efficiency.

The same comments can be made for the LTE antenna presented in Sec. 3. The measured s-parameter and total efficiency including the user effects can be seen respectively in Fig. 21 and Fig. 22. With the introduction of the hand and head, a shift in the resonance frequency can be seen towards the lower frequencies. Again, with the casing, a slightly positive effect is observed on the efficiency

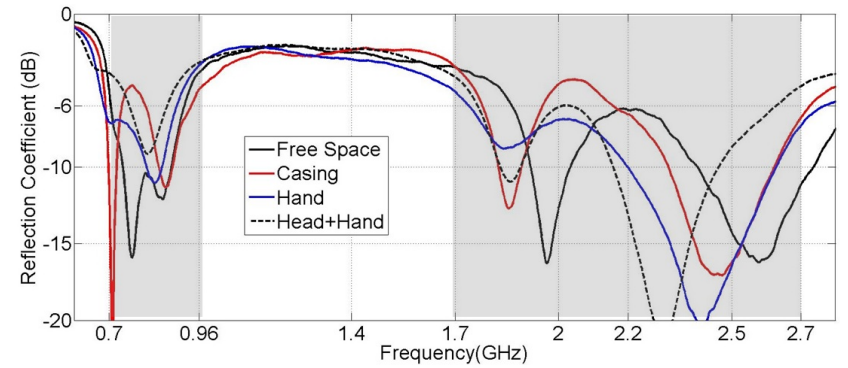

Fig. 21. Measured reflection coefficient of the LTE antenna taking into account user effects.

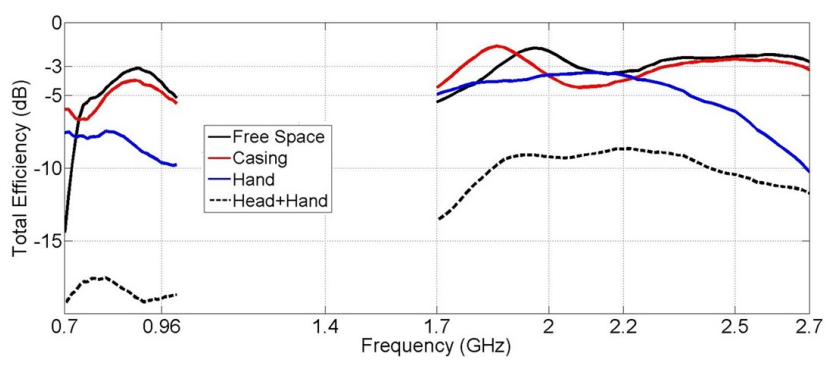

Fig. 22. Measured total efficiency of the LTE antenna taking into account user effects.

through dielectric loading. The resonance created by the parasitic strip around $700 \mathrm{MHz}$ (which is shifted down) increases the radiation efficiency and thus the total efficiency in the lower part of the LB (700-730 MHz). When the hand phantom is taken into account, the total efficiency is approximately $-7 /-10 \mathrm{~dB}$ which is equivalent as before. The direction of the main radiation is again seen away from the hand. When the head is also taken into account, further decrease is observed in the measured efficiency but in this case, the degradation is stronger in LB and smaller in HB.

\section{Conclusion}

Two antenna designs have been proposed for mobile terminals, realized using LDS technology. Both antennas consist of a driven strip which is exciting a parasitic strip connected to the ground plane of the PCB. The first antenna can cover $700-960 \mathrm{MHz}$ and $1.7-2.2 \mathrm{GHz}$, whereas the second antenna is improved in order to cover the whole 1.7-2.7 GHz frequency range in addition to the LB. These antennas have been designed in free space when optimizing reflection coefficient and total efficiency. The s-parameter and total efficiency of the fabricated prototypes were measured to validate the simulation results. Finally, to investigate the effect of the user hand and head as a basis for future study, the measurements were repeated using a dielectric casing and hand \& head phantoms. Further work will concentrate on designing antennas robust to the user effects in terms of matching. For instance, the BP versus frequency graph could be used in a different manner. Also, the observation of the evolution of the input impedance of the antenna when the user is taken into account could lead to a different approach for the antenna design in free space. Finally, a robust MN compensation versus the user interaction could be also studied. 


\section{Acknowledgments}

The authors would like to thank Orange Labs La Turbie and especially Frédéric Devillers and Patrice Brachat for their help during the measurements of the prototypes.

\section{References}

[1] RAPPAPORT, T.S., SUN, S., MAYZUS, R., ZHAO, H., AZAR, Y., WANG, K., WONG, G.N., SCHULZ, J.K., SAMIMI, M., GUTIERREZ, F. Millimeter wave mobile communications for 5G cellular: It will work!. IEEE Access, 2013, vol. 1, p. 335-349. DOI: 10.1109/ACCESS.2013.2260813

[2] VILLANEN, J., ICHELN, C., VAINIKAINEN, P. A coupling element-based quad-band antenna structure for mobile terminals. Microwave and Optical Technology Letters, June 2007, vol. 49, no. 6 , p. $1277-1282$. DOI: $10.1002 /$ mop. 22463

[3] ANDUJAR, A., ANGUERA, J., PUENTE, C. Ground plane boosters as a compact antenna technology for wireless handheld devices. IEEE Transactions on Antennas and Propagation, 2011, vol. 59, no. 5, p. 1668-1677. DOI: 10.1109/TAP.2011.2122299

[4] VALKONEN, R., ILVONEN, J., VAINIKAINEN P. Naturally non-selective handset antennas with good robustness against impedance mistuning. In Proceedings of the 6th European Conference on Antennas and Propagation (EuCAP). Prague (Czech Republic), March 2012, p. 796-800. DOI: 10.1109/EuCAP.2012.6206473

[5] VALKONEN, R., ILVONEN, J., ICHELN, C., VAINIKAINEN, $\mathrm{P}$. Inherently non-resonant multi-band mobile terminal antenna. IET Electronics Letters, 2013, vol. 49, no. 1, p. 11-13. DOI: $10.1049 / \mathrm{el} .2012 .3427$

[6] CHU, F.H., WONG, K.L. Internal coupled-fed loop antenna integrated with notched ground plane for wireless wide area network operation in the mobile handset. Microwave and Optical Technology Letters, March 2012, vol. 54, no. 3, p. 599-605. DOI: $10.1002 /$ mop. 26620

[7] YANG, C.W., JUNG, Y.B., JUNG, C.W. Octaband internal antenna for $4 \mathrm{G}$ mobile handset. IEEE Antennas and Wireless Propagation Letters, 2011, vol. 10, p. 817-819. DOI: 10.1109/LAWP.2011.2164049

[8] CHEN, S.C., WONG, K.L., Wideband monopole antenna coupled with a chip-inductor-loaded shorted strip for LTE/WWAN mobile handset. Microwave and Optical Technology Letters, June 2011, vol. 53, no. 6, p. 1293-1298. DOI: $10.1002 /$ mop. 25977

[9] CHEN, S.C., WONG, K.L. Small-size 11-band LTE/WWAN/WLAN internal mobile phone antenna. Microwave and Optical Technology Letters, November 2010, vol. 52, no. 11, p. 2603-2608. DOI: $10.1002 / \mathrm{mop} .25526$

[10] CHU, F.H., WONG, K.L. On-board small-size printed LTE/WWAN mobile handset antenna closely integrated with system ground plane. Microwave and Optical Technology Letters, June 2011 , vol. 53 , no. 6, p. 1336-1343. DOI: 10.1002/mop.25961

[11] LEE, C.T., WONG, K.L. Planar monopole with a coupling feed and an inductive shorting strip for LTE/GSM/UMTS operation in the mobile phone. IEEE Transactions on Antennas and Propagation, July 2010, vol. 58, no. 7, p. 2479-2483. DOI: 10.1109/TAP.2010.2048878

[12] WONG, K.L., TU, M.F., WU, T.Y., LI, W.Y. Small-size coupledfed printed PIFA for internal eight-band LTE/GSM/UMTS mobile phone antenna. Microwave and Optical Technology Letters,
September 2010, vol. 52, no. 9, p. 2123-2128. DOI: $10.1002 /$ mop. 25387

[13] WONG, K.L., CHEN, W.Y., WU, C.Y., LI, W.Y. Small-size internal eight-band LTE/WWAN mobile phone antenna with internal distributed LC matching circuit. Microwave and Optical Technology Letters, October 2010, vol. 52, no. 10, p. 2244-2250. DOI: $10.1002 / \mathrm{mop} .25431$

[14] LANXESS. POCAN DP T 7140 LDS 000000 (datasheet). 3 pages. [Online] Cited 2016-08-01. Available at: https://techcenter.lanxess.com/scp/americas/en/products/datasheet/ LANXESS_Pocan_DP_T_7140_LDS_000000_ISO_EN.pdf?docI $\mathrm{d}=12449715$

[15] LPKF. Three-Dimensional Circuits LPKF LDS: Laser Direct Structuring for $3 D$ Molded Interconnect Devices. 16 pages. [Online] Cited 2016-08-01. Available at: http://www.lpkf.com/_mediafiles/1797-lpkf-laser-directstructuring-en.pdf

[16] SONNERAT, F., PILARD, R., GIANESELLO, F., LE PENNEC, F., PERSON, C., GLORIA, D., BRACHAT, P., LUXEY, C. Innovative $4 \mathrm{G}$ mobile phone LDS antenna module using plastronics integration scheme. In Proceedings of the IEEE Antennas and Propagation Conference (AP-S). Orlando (USA), July 2013. DOI: 10.1109/APS.2013.6711767

[17] Optenni Lab. General Website. [Online] Cited 2016-08-01. Available at: https://www.optenni.com/

\section{About the Authors ...}

Aykut CIHANGIR was born in Ankara, Turkey in 1985. He received his Bachelor's degree from the Electrical and Electronics Engineering Department, Middle East Technical University (METU) in Ankara in 2007. From 2007 to 2011, he worked in the aerospace industry as a Communication Systems Design Engineer in Unmanned Aerial Vehicle projects. At the same period until 2010, he completed his Master's Degree study in METU about radiation characteristics of tapered slot antennas in millimeter-wave frequencies. From 2011 to 2014, he did his PhD study in University of Nice-Sophia Antipolis (UNSA), France on electrically small antennas and matching networks for $4 \mathrm{G}$ mobile terminals and obtained his $\mathrm{PhD}$ degree in 2014. He has then worked as a Post-Doctoral researcher in UNSA in the EpOC team. His research interests include electrically small antennas, matching networks and mobile terminal antennas.

Florence SONNERAT received the B.S. and M.S. degree in Electronics Engineering from PHELMA (School of Engineering in Physics, Applied Physics, Electronics \& Materials Science), Grenoble, France in 2010 and the Ph.D. degrees in Electrical Engineering from Telecom Bretagne, Brest, France, in 2013. Until mid 2015, she worked in CEA-LETI (France) where she was in charge of RF passive components characterization. She then worked for Radiall (France) for one year and designed RF connectors for high data rates applications. Since 2016, she works for STMicroelectronics (France) on RF passive components and electromagnetic devices modeling. She authored and coauthored about fifteen referred journal and conference technical articles. 
Frédéric GIANESELLO received the B.S. and M.S. degree in Electronics Engineering from Institut national polytechnique de Grenoble (Grenoble, France) in 2003 and the Ph.D. degrees in Electrical Engineering from the Joseph Fourier University (Grenoble, France) in 2006. Dr Gianesello has authored and coauthored more than 110 refereed journal and conference technical articles. He has served on the TPC for the International SOI Conference from 2009 up to 2011 and he is currently serving on the TPC for the Loughborough Antennas and Propagation Conference (LAPC). Dr Gianesello is currently working for STMicroelecetronics in Crolles (France) where he leads the team responsible for the development of electromagnetic devices (inductor, balun, transmission line, antenna, ...) integrated on advanced RF CMOS/BiMOS (down to $14 \mathrm{~nm}$ ), Silicon Photonics and advanced packaging technologies (3D Integration, FOWLP, ...).

Daniel GLORIA received the engineering degree in Electronics from the Ecole Nationale Supérieure d'Electronique et de Radioélectricité, Grenoble, France, in 1995 and the M.S.E.E. degree in optics, optoelectronics and microwave design systems from the Institut National de Grenoble (INPG), Grenoble, France. He spent two years, from 1995 to 1997, in ALCATEL Bell Network System Labs, Charleroi, Belgium, as an RF Designer Engineer and was involved in the development of the Cablephone RF front end and its integration in hybrid-fiber-coax telecommunication networks. Since 1997, he has been working for ST Microelectronics, Technology R\&D Crolles, TPS Laboratory, where he is in charge of HF characterization and RF passive modeling group. His interests are in the optimization of active and passive devices for HF applications in $\mathrm{Bi}$ CMOS and CMOS advanced technologies.

Cyril LUXEY (corresponding author) was born in Nice, France in 1971. He received the Ph.D. degree in Electrical Engineering (1999) with honors, from the University NiceSophia Antipolis, France. During his thesis, he worked on printed leaky-wave antennas, quasi-optical mixers and retrodirective transponders. From 2000 to 2002, he was with Alcatel, Mobile Phone Division, Colombes, France, where he was involved in the design and integration of internal antennas for commercial mobile phones. In 2003, he was recruited as an Associate Professor at the Polytech- nic school of the University Nice Sophia-Antipolis. Since 2009, he is a Full Professor at the IUT Réseaux et Télécoms in Sophia-Antipolis. He is doing his research in the EpOC laboratory as the vice-deputy of this lab. From October 2010 to December 2015, he was appointed as a Junior Member of the Institut Universitaire de France (IUF) institution for five years. His current research interests include the design and measurement of millimeter-wave antennas, antennas-in-package, plastic lenses, organic modules and silicon photonics solutions for 60, 120 and $240 \mathrm{GHz}$ frequency bands. Also, he collaborates with Berkeley Wireless Research Center and Stanford on mmwave front-end transceivers at mm-wave frequencies. He also works on electrically small antennas, multi-antenna systems for diversity and MIMO techniques. Cyril Luxey is an associate editor for IEEE Antennas and Wireless Propagation Letters, a reviewer for the IEEE Transactions on Antennas and Propagation, the IEEE Antennas and Wireless Propagation Letters, the IEEE Transactions on Microwave Theory and Techniques, the IEEE Microwave and Wireless Conference Letters, the IET Electronics Letters, the IET Microwave Antennas and Propagation journals and several European and US conferences in the field of microwave, microelectronics and antennas. Cyril Luxey and his students received the H.W. Wheeler Award of the IEEE Antennas and Propagation Society for the best application paper of the year 2006. Cyril Luxey is also the corecipient of the Jack Kilby Award 2013 of the ISSCC conference. He is the co-recipient of the best paper of the EUCAP2007 conference, the best-paper award of the International Workshop on Antenna Technology (iWAT2009), the best paper award at LAPC 2012, the best student paper at LAPC 2013 ( $3^{\text {rd }}$ place), the best paper of the ICEAA 2014 conference and the best paper of the innovation contest of the iWEM 2014 conference ( $2^{\text {nd }}$ place). Cyril Luxey has authored or co-authored more than 300 papers in refereed journals, in international and national conferences and as book chapters. He has given more than 10 invited talks. Cyril Luxey has been the general chair of the Loughborough Antennas and Propagation Conference 2011, the award and grant chair of EuCAP 2012 and the invited paper co-chair of EuCAP 2013. He will be the TPC chair of the EuCAP 2017 conference in Paris. Since 2015, he is a member of the IEEE AP-S Education committee. 